\title{
The costoclavicular syndrome: a 'new cause'
}

\author{
M DE SILVA
}

From the Department of Rheumatology, Prince Charles Hospital, Merthyr Tydfil, Mid-Glamorgan

SUMMARY Costoclavicular compression in obese, heavy breasted, middle aged or elderly women $\vec{\circ}$ from tight, narrow brassiere straps is a common cause of neck, shoulder, and arm pain. Clues to $\overrightarrow{\vec{\omega}}$ diagnosis and simple treatment measures, including the use of a shoulder pad, are described.

Key words: neck-shoulder-arm pain.

Chronic neck, shoulder, and arm pain is a common symptom complex for which many patients attend their general practitioners and a variety of hospital outpatient clinics.

The socioeconomic consequences of chronic backache are well recognised, but similar information on chronic neck, shoulder, and arm pain is scanty. Nevertheless, the cost in lost working hours and the demand on medical time must be considerable.

This paper describes a common, poorly understood, and hitherto undescribed cause of this condition. Awareness of its existence and simple remedial measures will reduce morbidity and the demand on limited resources.

\section{A ETIOLOGY}

The costoclavicular passage is one of three passages

Accepted for publication 13 May 1986.

Correspondence to Dr M De Silva.

that consitute the thoracic outlet; the others are the $\overrightarrow{\vec{A}}$ superior thoracic outlet and the costoscalene 0 hiatus. ${ }^{1}$ The neurovascular bundle is vulnerable to $\sigma$ compression in each of these situations. The costo- $\frac{}{2}$ clavicular passage is formed by the clavicle antero- laterally, the first rib medially, and the scapula posteriorly (Fig. 1).

The costoclavicular syndrome was first described 3 in soldiers with loaded knapsacks, who developed pain, numbness, and fatigueability of the arms $8 s \overrightarrow{0}$ they stood at attention. ${ }^{2}$ The mechanisms of cou pression involved downward movement of the cla icle against the first rib, with a resultant tendency shearing of the neurovascular bundle. This same mechanism is thought to explain subclavian vein thrombosis often precipitated by prolonged heavy음 exercise of the upper extremities-the Paget-o Schroetter syndrome.

A similar mechanism operates in usually obese,

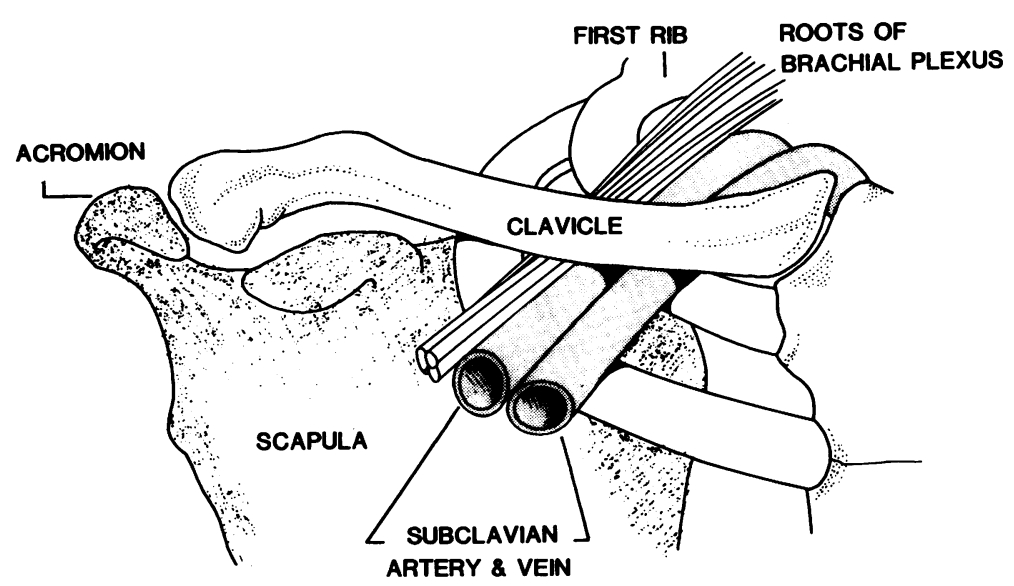

Fig. 1 Schematic diagram of the anatomy of the thoracic outlet. 
middle aged or elderly women. Tight, narrow brassiere straps supporting heavy breasts cut into the soft tissues around the shoulders and exert direct downward pressure on the clavicles, usually around the junction of the mid and lateral thirds. A scissoring action of the clavicle against the first rib narrows the costoclavicular passage and shears the neurovascular bundle. Subluxation of the lateral end of the clavicle relative to the acromial process of the scapula also results. Subjects have a tendency to stoop and be 'round shouldered', developed as an involuntary measure to relieve pressure on their shoulders. This further narrows the costoclavicular passage by pushing the scapula forwards.

PRESENTING COMPLAINTS

Pain or ache sometimes accompanied by stiffness in the neck and shoulders, pain, paraesthesiae, and fatigueability of the upper limbs are the main presenting complaints. Symptoms are usually bilateral, though more pronounced on the dominant side. They are aggravated by work and exercise, particularly carrying heavy shopping bags. Symptoms are relieved by rest and sleep, are minimal or absent in the morning, and become pronounced as the day progresses. Patients occasionally complain of puffy blue hands.

DIAGNOSIS

A high degree of suspicion, particularly in the vulnerable group, i.e., obese, heavy breasted, middle aged or elderly women, is essential. The average patient has had symptoms for several months or years and has attended several different clinics, e.g., accident, orthopaedics, neurology, rheumatology. She has had no relief from a variety of tablet and physical treatments and is labelled as having cervical spondylosis or brachialgia. The patient usually appears with a cervical collar, either in situ or in a bag.

EX A M I N A T I O N

The most important clues to diagnosis are the deep grooves on both shoulders where tight, narrow bra straps have cut deeply into the underlying soft tissues (Figs 2-4). Direct downward pressure with a forefinger in the groove reproduces symptoms. Invariably, there is tenderness over the acromioclavicular joint. Movements of the neck and shoulder are free. The former are painless, but shoulder movements may be slightly painful because of osteoarthritis of the acromioclavicular joint. There is no muscle wasting or weakness.

Paraesthesiae when present are likely to involve the thumb and all fingers and sometimes the whole limb. They differ from the paraesthesiae of carpal tunnel compression in their distribution and timing. Phalen's and Tinel's signs are absent. Some patients complain of puffy blue hands. They lack the classic colour changes of Raynaud's phenomenon and are unaffected by cold. The normal appearance of the hand in the costoclavicular syndrome helps to differentiate it from the shoulder-hand syndrome, where it is shiny, swollen, warm, and hyperaesthetic.

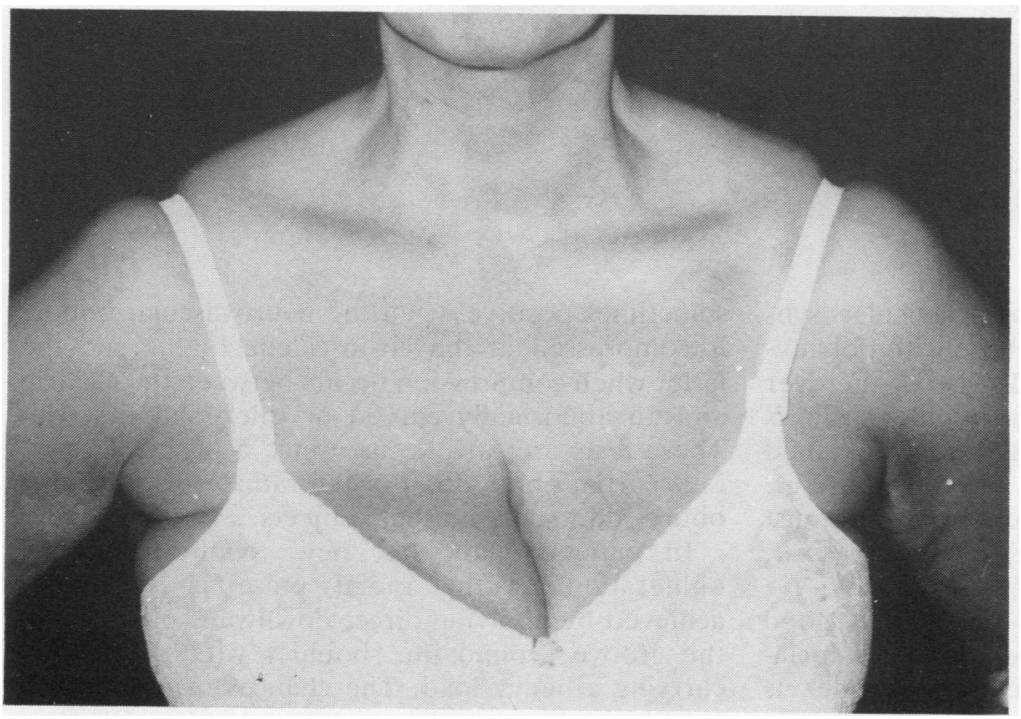

Fig. 2 Brassiere straps cutting deeply into the underlying soft tissue 


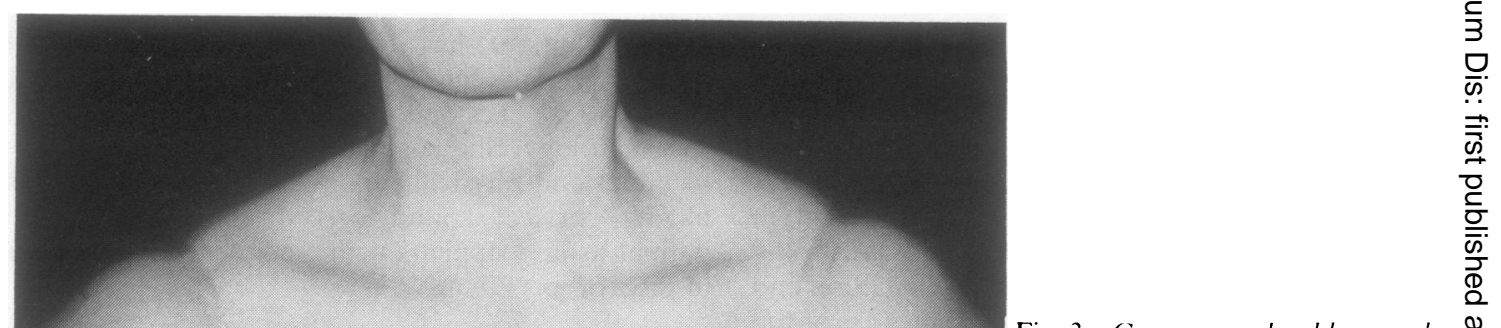

Fig. 3 Grooves on shoulders can be के

clearly seen.

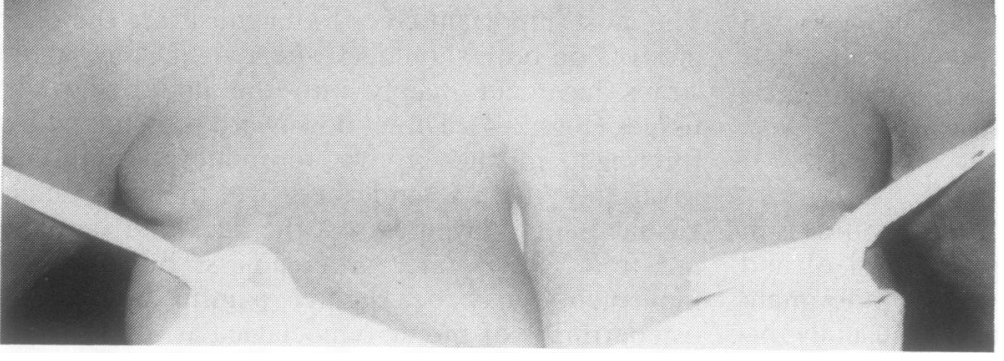

Fig. 4 Grooves on shoulders can

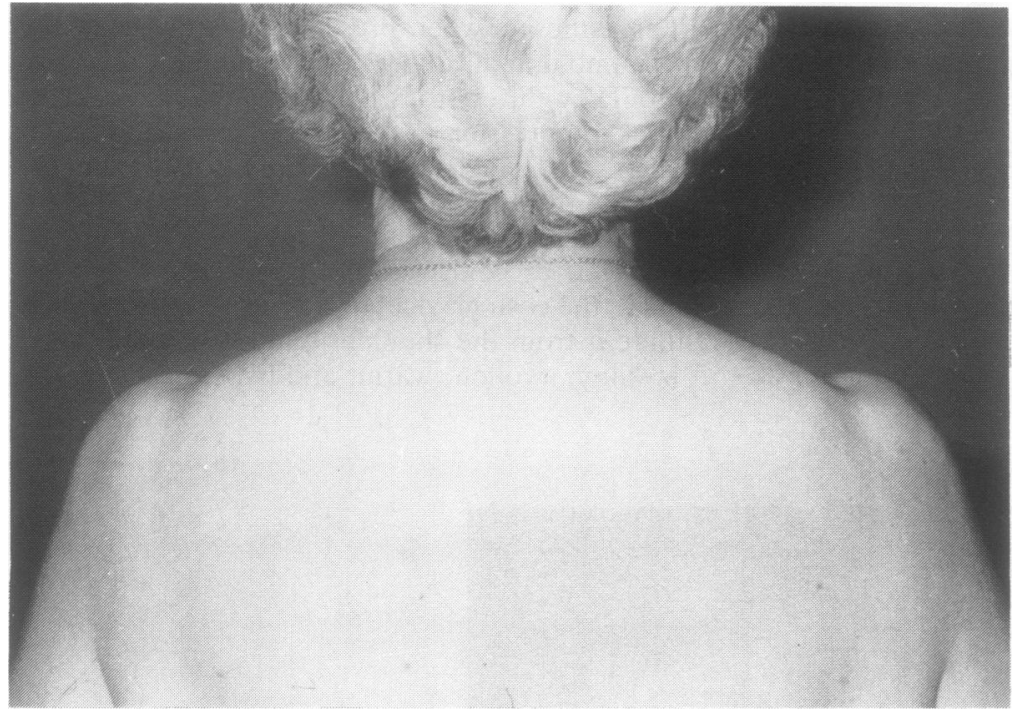

be clearly seen.

Patients with this form of costoclavicular syndrome are usually younger than those with polymyalgia rheumatica, customarily a disease of the over sixties. In polymyalgia rheumatica shoulder stiffness is prominent and bilateral and may be accompanied by symptoms of systemic upset or arteritis, e.g., visual disturbances, headache, etc. Great care must be taken to exclude the other causes of neck, shoulder, and arm pain (Table 1).

Radial pulses are present, equal, and of good volume. Both Adson's manoeuvre and the costoclavicular manoeuvre are negative. The former is sometimes positive when the neurovascular bundle is compressed in the costoscalene hiatus and the latter when compression occurs between the clavicle and an abnormally curved or thickened first rib. N These tests are of little use since a positive result, $N$ i.e., partial obliteration of the radial pulse, may be obtained in some normal subjects.

In patients with this 'new' syndrome partial obliteration of the radial pulse is sometimes achieved by applying direct downward pressure on the groove around the shoulder with the patient carrying a heavy load. The chances of obtaining a 
Table 1 Causes of chronic neck, shoulder, and arm pain

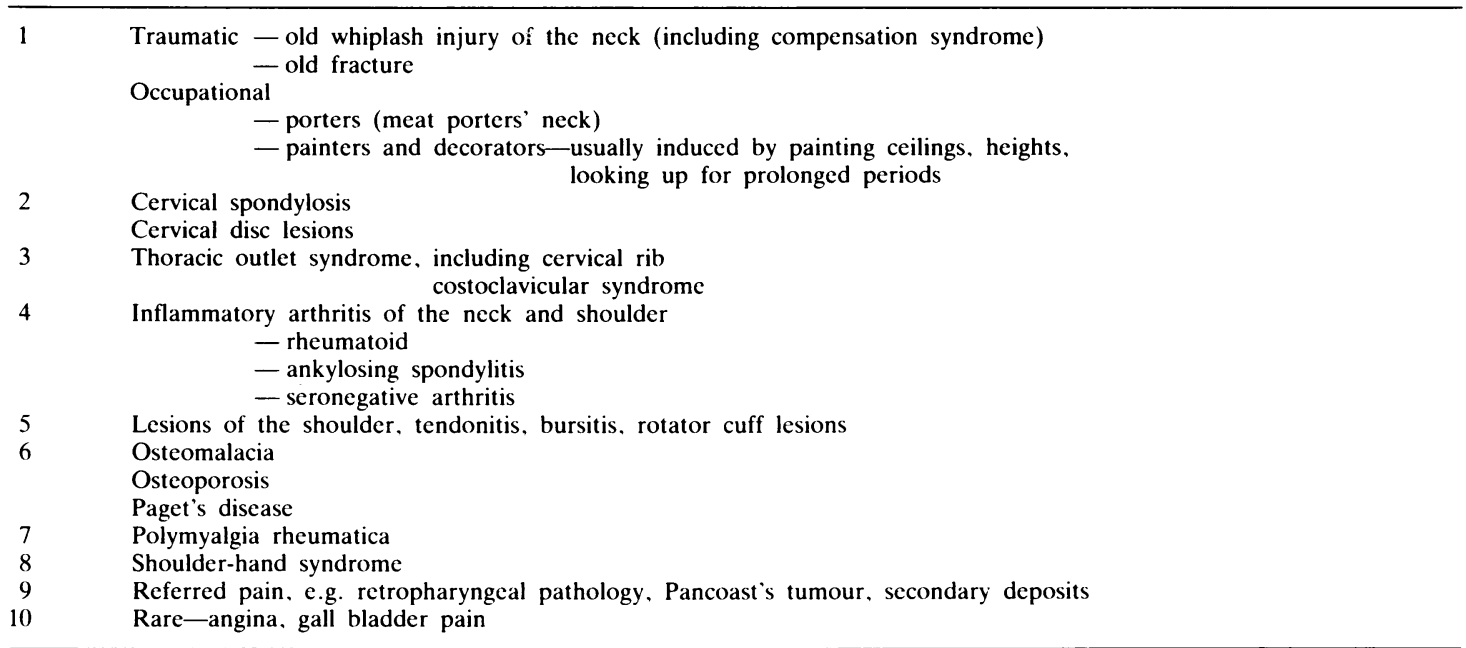

positive test are enhanced by fatigue, which sets in after the patient has carried the load for $10-15$ minutes. This manoeuvre is unnecessary, cumbersome, rarely practicable in a busy outpatient clinic, and above all uncomfortable for the patient.

\section{NVESTIGATIONS}

Patients have often been extensively investigated. No abnormalities are detected on routine blood tests, full blood count, erythrocyte sedimentation rate, and immunological tests for inflammatory arthritis. The long duration of symptoms, normal blood tests, and $x$ rays usually showing no more than minimal degenerative change of the cervical spine and acromioclavicular joint exclude most other conditions to be considered in the differential diagnosis.

The approximation of the clavicle to the first rib that occurs when direct downward pressure is applied on the grooves of the shoulder or on loading is difficult to demonstrate radiologically. This is due to technical difficulties in obtaining radiographs of comparable quality and of placing markers on the first rib. The subluxation of the acromioclavicular joint may sometimes be seen.

M A N A G E M EN T

A clear explanation of the underlying mechanism for the symptoms, preferably with the aid of a plastic model, is most important. The next step is the elimination of the cause of symptoms. Patients must be advised to wear either strapless brassieres or

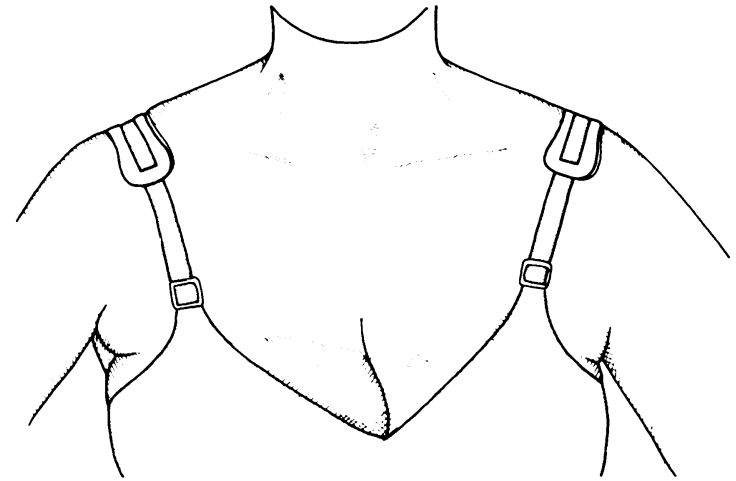

Fig. 5 Diagram showing shoulder pads in place-threaded through broad brassiere straps.

brassieres with broad straps with a pad threaded through the strap and placed in position on the shoulders (Fig. 5). These measures help to distribute the downward force exerted by the straps. The straps must not be too tight. Patients must avoid carrying heavy shopping bags. The use of a shopping trolley solves this problem. Attention to posture directed at avoiding stooping and shoulder girdle exercises helps. Weight reduction though seldom resulting in significant reduction in the weight of the breasts helps, if only to increase the sense of general wellbeing.

Pain and tenderness in the acromioclavicular joint usually respond to a reduction of the distracting 
forces on the joint produced by the measures outlined. Some may need treatment with local ice or heat and simple analgesics. Occasionally an injection of corticosteroid and local anaesthetic into the acromioclavicular joint may be required. In extreme cases surgical reduction of breast size by mammoplasty produces excellent results.

My thanks to Mrs Elaine Thomas, secretary, Postgraduate Centre, Prince Charles Hospital, Merthyr Tydfil, for typing the manuscript and to Mrs Janice Sharpe of the Department of Medical Illustration. University Hospital of Wales, Cardiff, for Figs 1 and 5.

\section{References}

1 Pollak E W. Surgical anatomy of the thoracic outlet syndrome Surg Gynecol Obstet 1980; 150: 97-103.

2 Falconer M, Weddel G. Costoclavicular compression of the subclavicular artery and vein. Lancet 1943; ii: 539-44.

3 Hughes E S R. Collective review; venous obstruction in upper extremity (Paget-Schroetter's syndrome): review of 320 cases. Surg Gynecol Obstet 1949: 88 (suppl): 89-127. 